OPEN ACCESS

Edited by:

Daniele Santi,

University of Modena and Reggio

Emilia, Italy

Reviewed by:

Ljiliana Marina

University of Belgrade, Serbia Ludwig Wildt,

Innsbruck Medical University, Austria

*Correspondence: Xiaofeng Yang yxf73@163.com

Specialty section: This article was submitted to

Reproduction

a section of the journal

Frontiers in Endocrinology

Received: 12 December 2019 Accepted: 10 March 2020

Published: 31 March 2020

Citation:

Wang L, LV S, Li F, Yu X, Bai E and Yang X (2020) Vitamin D Deficiency Is

Associated With Metabolic Risk

Factors in Women With Polycystic

Ovary Syndrome: A Cross-Sectional

Study in Shaanxi China.

Front. Endocrinol. 11:171.

doi: 10.3389/fendo.2020.00171

\section{Vitamin D Deficiency Is Associated With Metabolic Risk Factors in Women With Polycystic Ovary Syndrome: A Cross-Sectional Study in Shaanxi China}

\author{
Li Wang, Shulan Lv, Fen Li, Xuewen Yu, E. Bai and Xiaofeng Yang* \\ Department of Obstetrics and Gynecology, The First Affiliated Hospital of Xi'an Jiaotong University, Xi'an, China
}

Background: Polycystic ovary syndrome (PCOS) is a common endocrine disorder in women at reproductive age, which is characterized by obesity, hyperandrogenemia, and insulin resistance (IR). This study aimed to investigate the vitamin D status, and analyze the relationship between vitamin D deficiency and metabolic risk factors in PCOS women in Shaanxi China.

Methods: A cross-sectional study included 169 women diagnosed with PCOS and 114 control women without PCOS. The serum 25(OH)D and metabolic markers were measured. Vitamin D deficiency was defined as serum $25(\mathrm{OH})$ D concentration less than $20 \mathrm{ng} / \mathrm{mL}$. The primary outcome was the difference in vitamin D status between the PCOS and control groups, the secondary outcomes were correlations between serum 25(OH)D concentration and metabolic risk factors in women with PCOS.

Results: The serum 25(OH)D concentration was significantly lower in women with PCOS than in controls $(P<0.05)$, and the prevalence rates of $25(\mathrm{OH}) \mathrm{D}$ deficiency and insufficiency were significantly higher in women with PCOS than in controls $(P<$ 0.05). The serum $25(\mathrm{OH}) \mathrm{D}$ concentration was significantly lower in PCOS women with obesity or $\mathbb{I R}$ than in women without obesity or $\mathbb{I R}(P<0.05)$, and the prevalence of $25(\mathrm{OH}) \mathrm{D}$ deficiency in PCOS women with obesity or IR was significantly higher than in women without obesity or $\mathbb{R}(P<0.05)$. Serum $25(\mathrm{OH}) \mathrm{D}$ concentration was significantly negatively correlated with body mass index (BMI), waist-to-hip ratio (WHR), fasting insulin, homeostasis model assessment of insulin resistance (HOMA$I R$ ), total cholesterol, low-density lipoprotein cholesterol (LDL-C), and high-sensitivity C-reactive protein (hs-CRP) $(P<0.05)$. In comparison, serum 25(OH)D concentration was significantly positively correlated with high-density lipoprotein cholesterol (HDL-C) $(P<0.05)$. Increased $\mathrm{BMI}$ and WHR, high levels of fasting insulin, HOMA-IR, total cholesterol, LDL-C and hs-CRP were regarded as risk factors, but high level of HDL-C was considered to be protective factor of vitamin D deficiency in PCOS women. 


\begin{abstract}
Conclusions: Vitamin D deficiency is prevalent in PCOS women in Shaanxi China, especially in those with obesity and IR. The serum 25(OH)D level was correlated with metabolic risk factors in PCOS women. Multi-center randomized controlled trials with large sample sizes are needed to probe the metabolic effect of vitamin D supplementation in PCOS women.
\end{abstract}

Keywords: vitamin D deficiency, metabolic disturbances, polycystic ovary syndrome, obesity, insulin resistance, Shaanxi China

\section{INTRODUCTION}

Polycystic ovary syndrome (PCOS) is a common endocrine disorder in women at reproductive age, the prevalence of PCOS ranging from 9 to $18 \%(1,2)$. Obesity, hyperandrogenemia, and insulin resistance (IR) are prevalent characteristics of PCOS (3). Studies have confirmed that PCOS is related to type 2 diabetes mellitus (T2DM), cardiovascular disease and metabolic syndrome $(4,5)$. A meta-analysis showed that $30-40 \%$ of women diagnosed with PCOS accompany IR and compensatory hyperinsulinaemia, and that $\sim 10 \%$ of these women will develop T2DM (6). Furthermore, PCOS women were vulnerable to present with dyslipidaemia, such as higher concentrations of triglycerides (TGs) and low-density lipoprotein cholesterol (LDL-C), and lower high-density lipoprotein cholesterol (HDL-

C) than healthy women (7).

It is known that vitamin $\mathrm{D}$ regulate the skeletal growth and development and calcium/phosphorus metabolism. Nevertheless, vitamin D also is related to other diseases, including immune disorders (8), T2DM (9), cardiovascular disease (10), infectious diseases, and cancer (11). In recent years, the difference of vitamin D levels between PCOS women and healthy women, the relationship between vitamin $\mathrm{D}$ and metabolic factors in PCOS women has remained controversial. Some studies have shown that PCOS women had lower serum 25(OH)D concentration than healthy women, and vitamin D deficiency was associated with homeostasis model assessment of insulin resistance (HOMA-IR), hyperinsulinaemia, dyslipidaemia, and metabolic risk factors in patients with PCOS $(12,13)$. However, other scholars have found different results (14).

Vitamin D deficiency and PCOS are associated with metabolic disorders, but little is known about vitamin D status in women with PCOS in Shaanxi China. Therefore, this cross-sectional study aims to investigate vitamin D status and analyze the relationship between vitamin $\mathrm{D}$ deficiency and metabolic risk factors in women with PCOS.

\section{MATERIALS AND METHODS Study Design and Participants}

This survey was a cross-sectional study. A total of 169 women diagnosed with PCOS were recruited through random sampling from five different communities in the city of Xi'an in Shaanxi, China. Participants were selected from the gynecological clinic and women's health clinic in the
First Affiliated Hospital of Xi'an Jiaotong University from January 2018 to May 2019. One hundred and fourteen women without PCOS were selected as controls at the same time. The diagnostic criteria of PCOS we used were the modified Rotterdam criteria (15). Women with thyroid disease, congenital adrenal hyperplasia or autoimmune disease were excluded. The protocol was approved by the First Affiliated Hospital of Xi'an Jiaotong University Institutional Review Board.

\section{Outcome Measures}

A questionnaire was administered to each participant in a face-to-face interview. Basic information was collected, including age, body mass index (BMI), waist-to-hip ratio (WHR), and blood pressure. BMI $\geq 28.0 \mathrm{~kg} / \mathrm{m}^{2}$ was defined as obesity (16). All biochemical indicators were tested in our hospital including serum 25-hydroxyvitamin $\mathrm{D}[25(\mathrm{OH}) \mathrm{D}]$, follicle-stimulating hormone (FSH), luteinizing hormone $(\mathrm{LH})$, prolactin (PRL), testosterone (T), estradiol $\left(\mathrm{E}_{2}\right)$, progesterone $(\mathrm{P})$, blood glucose, insulin, blood lipids, and high-sensitivity C-reactive protein (hs-CRP). HOMA-IR was calculated to evaluate insulin resistance (IR). HOMA$\mathrm{IR}=$ [fasting glucose $(\mathrm{mmol} / \mathrm{L}) \times$ fasting insulin $(\mathrm{mIU} / \mathrm{L})] /$ 22.5. HOMA-IR > 2.5 was defined as IR (17). The serum $25(\mathrm{OH}) \mathrm{D}$ levels lower than $20 \mathrm{ng} / \mathrm{mL}$ were defined as vitamin D deficiency, and women with a concentration of $20 \sim 30 \mathrm{ng} / \mathrm{mL}$ were defined as vitamin D insufficiency. Serum $25(\mathrm{OH}) \mathrm{D}$ concentration of $30 \sim 50 \mathrm{ng} / \mathrm{mL}$ were defined as normal (18).

The primary outcome was the difference of vitamin D level between the PCOS and control groups. The secondary outcomes were correlations between serum $25(\mathrm{OH}) \mathrm{D}$ concentration and metabolic risk factors in PCOS women.

\section{Statistical Analysis}

Data in this study were analyzed using SPSS version 20.0. The continuous variables are presented as mean $\pm \mathrm{SD}$, which were performed by Student's $t$-test or variance analysis. The categorical parameters are displayed as numbers (\%), which were analyzed by chi-square test. Linear regression analysis was used to analyze the correlation of $25(\mathrm{OH}) \mathrm{D}$ concentration with metabolic parameters. The relationship between vitamin D deficiency and metabolic risk factors were analyzed by multinomial logistic regression analysis. $P<0.05$ was considered statistically significant. 


\section{RESULTS}

\section{Basic Characteristic of the Studied Groups}

Table 1 illustrates the baseline characteristics of women in the two groups. The BMI, WHR, and serum LH and T concentrations were significantly higher in women with PCOS

TABLE 1 | Baseline characteristics of women between the two groups.

\begin{tabular}{|c|c|c|c|}
\hline & $\begin{array}{l}\text { PCOS group } \\
(n=169)\end{array}$ & $\begin{array}{l}\text { Control group } \\
\quad(n=114)\end{array}$ & $P$-value ${ }^{a}$ \\
\hline Age (years) $^{\mathrm{b}}$ & $28.4 \pm 8.3$ & $27.2 \pm 7.9$ & 0.872 \\
\hline $\mathrm{BMI}\left(\mathrm{kg} / \mathrm{m}^{2}\right)^{\mathrm{b}}$ & $24.7 \pm 6.5$ & $21.8 \pm 5.6$ & 0.046 \\
\hline WHR & $0.9 \pm 0.3$ & $0.7 \pm 0.3$ & 0.048 \\
\hline Blood pressure ${ }^{\mathrm{b}}$ & & & 0.409 \\
\hline Systolic (mm Hg) & $115.6 \pm 14.6$ & $109.5 \pm 13.8$ & 0.122 \\
\hline Diastolic (mm Hg) & $81.2 \pm 9.3$ & $76.9 \pm 8.5$ & 0.346 \\
\hline Outdoor exercise ${ }^{c}$ & & & 0.302 \\
\hline Never & $51(30.2 \%)$ & $28(24.6 \%)$ & \\
\hline$\geq 1$ times daily & 118 (69.8\%) & 86 (75.4\%) & \\
\hline Marital status ${ }^{c}$ & & & 0.213 \\
\hline Single & 45 (26.6\%) & $23(20.2 \%)$ & \\
\hline Married & 124 (73.4\%) & 91 (79.8\%) & \\
\hline \multicolumn{4}{|l|}{ Basal concentration ${ }^{b}$} \\
\hline $\mathrm{FSH}(\mathrm{mlU} / \mathrm{mL})$ & $6.8 \pm 1.7$ & $7.4 \pm 1.8$ & 0.457 \\
\hline $\mathrm{LH}(\mathrm{mlU} / \mathrm{mL})$ & $12.5 \pm 3.2$ & $9.3 \pm 2.8$ & 0.032 \\
\hline $\mathrm{PRL}(\mathrm{ng} / \mathrm{mL})$ & $15.4 \pm 5.8$ & $13.5 \pm 4.2$ & 0.053 \\
\hline $\mathrm{E}_{2}(\mathrm{pmol} / \mathrm{L})$ & $89.1 \pm 16.4$ & $79.5 \pm 14.1$ & 0.179 \\
\hline $\mathrm{T}(\mathrm{nmol} / \mathrm{L})$ & $1.9 \pm 0.8$ & $1.4 \pm 0.6$ & 0.042 \\
\hline $\begin{array}{l}\text { Time of blood } \\
\text { detection }^{c}\end{array}$ & & & 0.701 \\
\hline Spring & $36(21.3 \%)$ & $29(25.4 \%)$ & \\
\hline Summer & 52 (30.8\%) & 34 (29.8\%) & \\
\hline Autumn & $43(25.4 \%)$ & 31 (27.2\%) & \\
\hline Winter & 38 (22.5\%) & 20 (17.5\%) & \\
\hline
\end{tabular}

${ }^{a}$ T-test or chi-square test.

${ }^{b}$ Data are presented as mean $\pm S D$.

${ }^{c}$ Data are presented as number(\%). than in controls $(P<0.05)$. No significant difference was found when comparing other baseline characteristics between the two groups $(P>0.05)$.

\section{Vitamin D Status Between PCOS Women and Controls}

The serum 25(OH)D concentration was significantly lower in PCOS women than in controls $(11.6 \pm 7.2$ vs. $18.9 \pm 8.4 \mathrm{ng} / \mathrm{mL}$, $P<0.05)$ (Figure 1A). In addition, the prevalence rates of $25(\mathrm{OH}) \mathrm{D}$ deficiency and insufficiency were significantly higher in women with PCOS than in controls $(54.4 \%$ vs. $37.7 \%, P<$ $0.01 ; 34.9 \%$ vs. $23.7 \%, P<0.05)$. Furthermore, the prevalence of normal 25(OH)D status in women with PCOS was significantly lower than that in controls $(10.7 \%$ vs. $38.6 \%, P<0.01)$ (Figure 1B).

The serum 25(OH)D concentration was significantly lower in PCOS women with obesity or IR than in women without obesity or IR $(8.9 \pm 3.7$ vs. $13.6 \pm 5.3 \mathrm{ng} / \mathrm{mL}, P<0.05 ; 7.2 \pm 2.9$ vs. $15.8 \pm 4.9 \mathrm{ng} / \mathrm{mL}, P<0.01$ ) (Figures $2 \mathrm{~A}, \mathrm{C}$ ). Additionally, the prevalence of 25(OH)D deficiency in PCOS women with obesity or IR was significantly higher than in women without obesity or IR $(57.4 \%$ vs. $40.0 \%, P<0.05 ; 64.4 \%$ vs. $35.3 \%, P<0.01)$ (Figures 2B,D).

Table 2 shows the metabolic parameters among different vitamin D statuses in women with PCOS. There were statistically significant differences in BMI, WHR, fasting insulin, HOMA-IR, total cholesterol, LDL-C, HDL-C, and hs-CRP among the three groups $(P<0.05)$. No significant difference was found when comparing fasting glucose and triglycerides among different groups $(P>0.05)$.

\section{Vitamin D and Metabolic Risk Factors in PCOS Women}

The data in Table 3 suggest that serum 25(OH)D concentration was significantly negatively correlated with BMI, WHR, fasting insulin, HOMA-IR, total cholesterol, LDL-C and hs-CRP $(P$ $<0.05)$. In comparison, serum $25(\mathrm{OH}) \mathrm{D}$ concentration was significantly positively correlated with HDL-C $(P<0.05)$. However, no significant correlation was found between serum
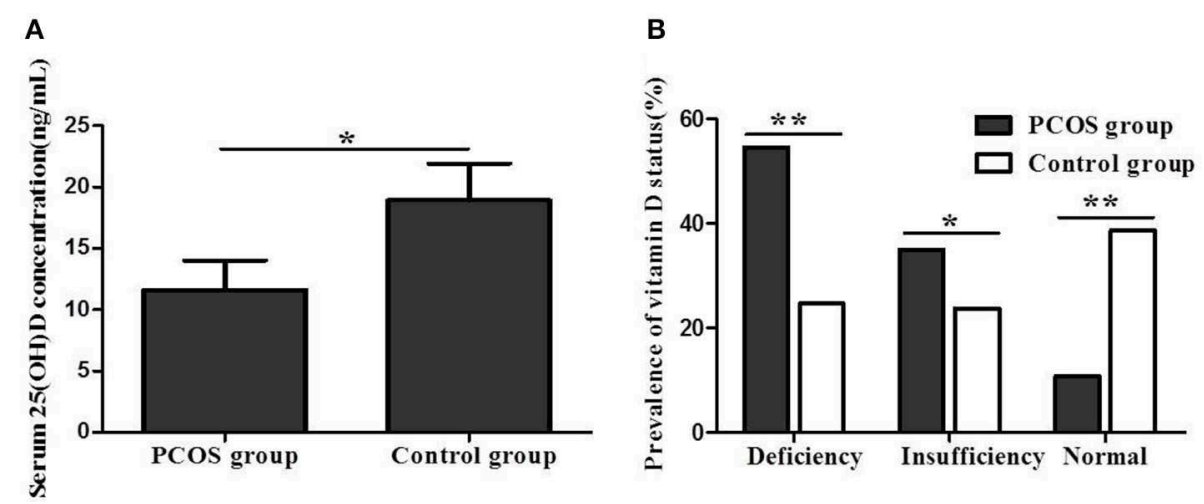

FIGURE 1 | (A) Serum 25(OH)D concentration between women with PCOS and control group. (B) Vitamin D status between women with PCOS and control group $\left({ }^{\star} P<0.05,{ }^{\star \star} P<0.01\right)$. 

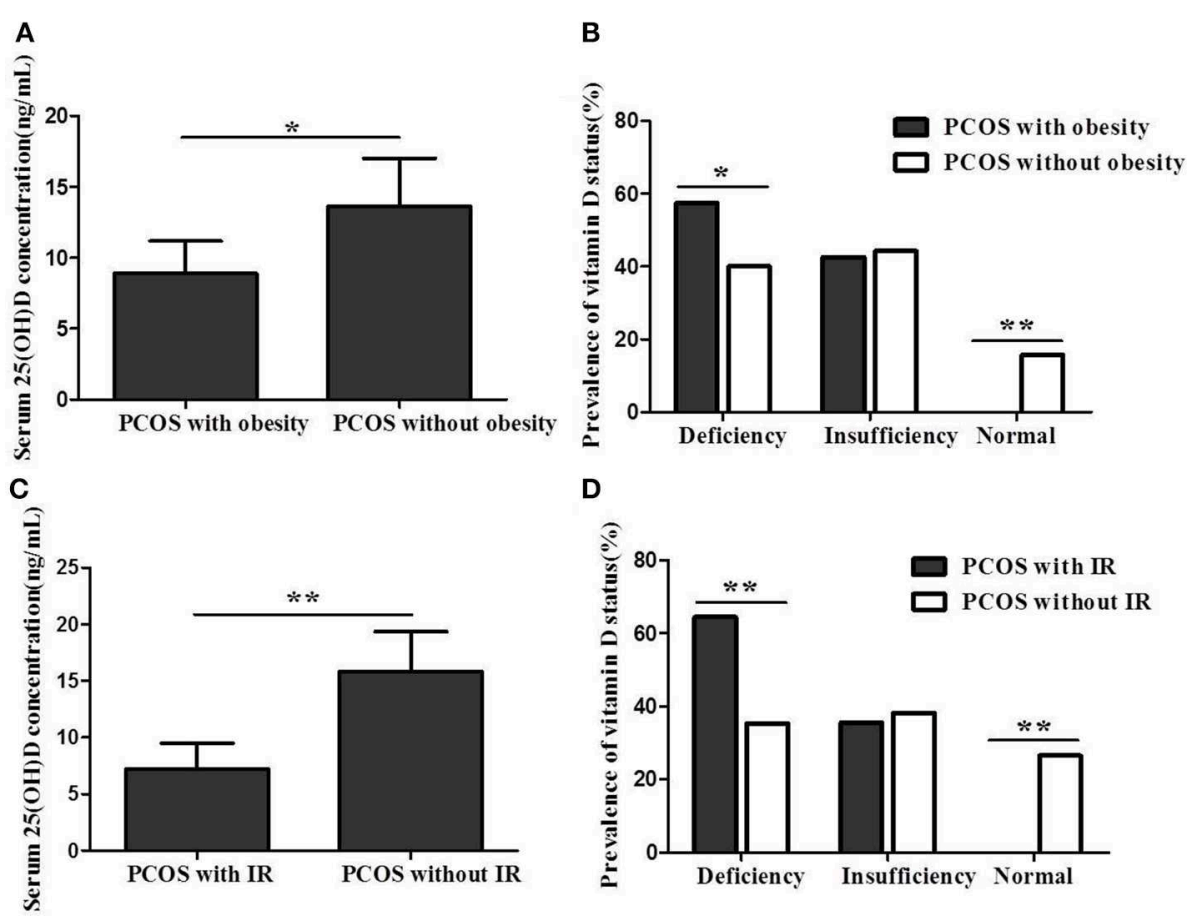

FIGURE 2 | (A) Serum 25(OH)D concentration between PCOS women with obesity and women without obesity. (B) Vitamin D status between PCOS women with obesity and women without obesity. (C) Serum 25(OH)D concentration between PCOS women with IR and women without IR. (D) Vitamin D status between PCOS women with IR and women without IR $\left({ }^{\star} P<0.05,{ }^{\star \star} P<0.01\right)$.

TABLE 2 | Metabolic parameters among different vitamin D status in women with PCOS.

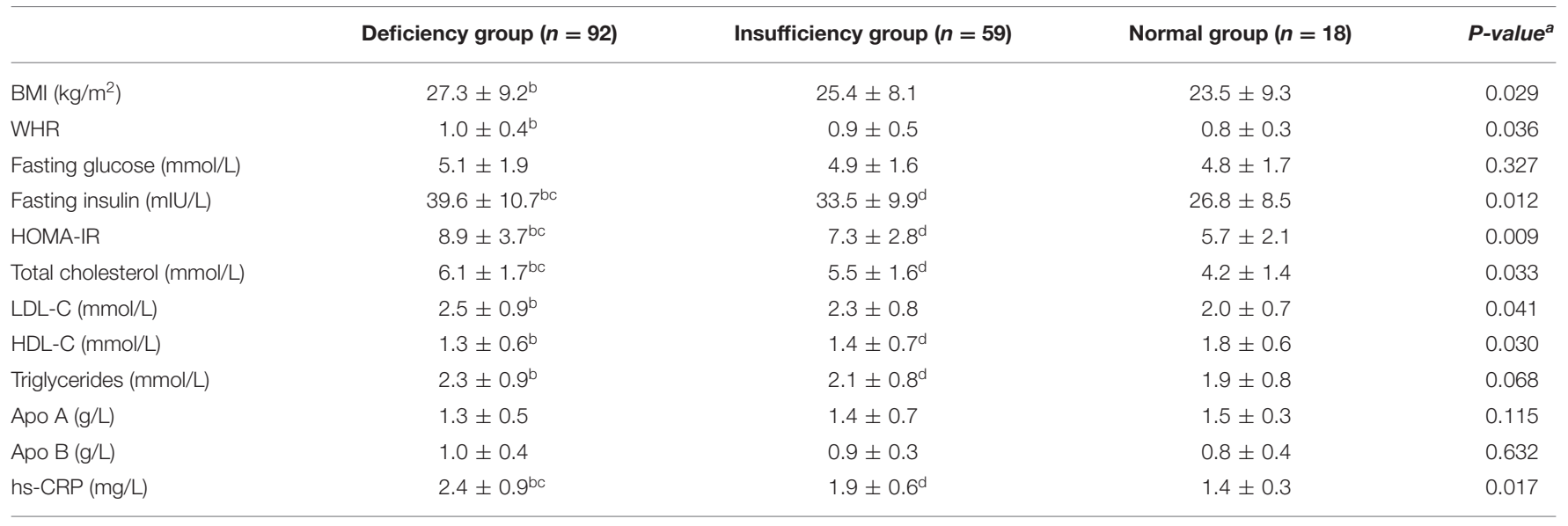

a Variance analysis.

${ }^{b}$ Deficiency group vs. Normal group, $P<0.05$.

${ }^{c}$ Deficiency group vs. Insufficiency group, $P<0.05$.

${ }^{d}$ Insufficiency group vs. Normal group, $P<0.05$.

25(OH)D concentration and age, fasting glucose, triglycerides, Apo A or Apo B $(P>0.05)$.

The data in Table 4 suggest that increased BMI and WHR, high levels of fasting insulin, HOMA-IR, total cholesterol, LDL-C and hs-CRP were regarded as risk factors of vitamin D deficiency in women with PCOS. Additionally, outdoor exercise $(\geq 1$ times daily) and high level of HDL-C were considered to be protective factors of vitamin D deficiency in PCOS women.

\section{DISCUSSION}

More than one billion children and adults are suffering from vitamin D deficiency, which is common worldwide (19). PCOS women manifest a relatively high prevalence of vitamin D deficiency than healthy women, and vitamin D deficiency is associated with ovulatory dysfunction, IR and hyperandrogenism (20). Thus, in recent years, many studies have been performed 
TABLE 3 | Correlation of serum 25(OH)D concentration with metabolic parameters in PCOS women.

\begin{tabular}{lcc}
\hline & $\boldsymbol{R}$ & $\boldsymbol{P}$-value \\
\hline Age (years) & -0.089 & 0.582 \\
BMI (kg/m²) & -0.581 & 0.016 \\
WHR & -0.424 & 0.032 \\
Fasting glucose (mmol/L) & -0.246 & 0.237 \\
Fasting insulin (mIU/L) & -0.624 & 0.003 \\
HOMA-IR & -0.635 & 0.001 \\
Total cholesterol (mmol/L) & -0.426 & 0.032 \\
LDL-C (mmol/L) & -0.474 & 0.022 \\
HDL-C (mmol/L) & 0.419 & 0.043 \\
Triglycerides (mmol/L) & -0.326 & 0.108 \\
Apo A (g/L) & -0.113 & 0.567 \\
Apo B (g/L) & -0.076 & 0.683 \\
hs-CRP (mg/L) & -0.539 & 0.028 \\
\hline
\end{tabular}

to analyze vitamin D status of PCOS women (21). Data from our study showed that the serum 25(OH)D concentration was significantly lower, and the prevalence rates of $25(\mathrm{OH}) \mathrm{D}$ deficiency and insufficiency were significantly higher in women with PCOS than in controls, the results is similar to previous study (22). It has been confirmed that PCOS women accompany vitamin D deficiency, and $67-85 \%$ of them had serum $25(\mathrm{OH}) \mathrm{D}$ level $<20 \mathrm{ng} / \mathrm{mL}$ (23).

Multiple observational studies have shown that low vitamin D concentration is associated with increased BMI, IR, testosterone and dehydroepiandrosterone sulfate (DHEAS) levels in women with PCOS (24), which was consistent with our study, where we showed that serum $25(\mathrm{OH}) \mathrm{D}$ concentration was significantly lower in PCOS women with obesity or IR than in women without obesity or IR. In addition, the prevalence of $25(\mathrm{OH}) \mathrm{D}$ deficiency in PCOS women with obesity or IR was significantly higher than in women without obesity or IR. Epidemiological studies have confirmed that vitamin $\mathrm{D}$ status was negatively associated with diabetes, which may be related to multiple factors. Research confirmed that $25(\mathrm{OH}) \mathrm{D}$ can increase the expression of insulin receptors, and inhibit the release of inflammatory cytokines that are proved to cause IR (25). Furthermore, it has been reported that vitamin $\mathrm{D}$ has an indirect useful function on insulin role through regulation of extracellular calcium (26). However, other researchers have revealed different results. $\mathrm{Ng}$ et al. demonstrated that there was no statistically significant correlation between vitamin $\mathrm{D}$ level and $\mathrm{BMI}, \mathrm{WHR}$, and metabolic parameters among PCOS women (14). This difference may be explained by differences in sample size, habits of the participants, place of residence, and season of detection of vitamin $\mathrm{D}$.

Concerning the lipid profile, this study demonstrated that serum $25(\mathrm{OH}) \mathrm{D}$ concentration in women with PCOS was significantly and negatively correlated with total cholesterol and LDL-C but was significantly positively correlated with HDL-C. This finding was concordant with the results of previous studies (27). It is well-known that cardiovascular disease is associated with high total cholesterol and LDL-C levels and low HDL$\mathrm{C}$ levels. Therefore, vitamin $\mathrm{D}$ supplementation may reduce
TABLE 4 | Multinomial logistic regression model of risk factors for vitamin D deficiency in women with PCOS.

\begin{tabular}{|c|c|c|c|}
\hline & $95.0 \% \mathrm{Cl}$ & OR & P-value \\
\hline \multicolumn{4}{|l|}{ Age (years) } \\
\hline$\leq 25$ & Reference & 1.00 & - \\
\hline $26-34$ & $0.48-1.91$ & 1.02 & 0.692 \\
\hline$\geq 35$ & $0.53-2.06$ & 1.13 & 0.583 \\
\hline \multicolumn{4}{|l|}{ BMI $\left(\mathrm{kg} / \mathrm{m}^{2}\right)$} \\
\hline$<25$ & Reference & 1.00 & - \\
\hline$\geq 25$ & $1.23-5.18$ & 2.53 & 0.011 \\
\hline \multicolumn{4}{|l|}{ WHR } \\
\hline$<0.85$ & Reference & 1.00 & - \\
\hline$\geq 0.85$ & $1.17-3.92$ & 2.17 & 0.019 \\
\hline \multicolumn{4}{|l|}{ Hypertension } \\
\hline No & Reference & 1.00 & - \\
\hline Yes & $0.29-2.97$ & 1.24 & 0.376 \\
\hline \multicolumn{4}{|c|}{ Outdoor exercise } \\
\hline Never & Reference & 1.00 & - \\
\hline$\geq 1$ times daily & $0.31-0.93$ & 0.49 & 0.032 \\
\hline \multicolumn{4}{|c|}{ Fasting glucose (mmol/L) } \\
\hline$<6.1$ & Reference & 1.00 & - \\
\hline$\geq 6.1$ & $0.57-2.03$ & 1.57 & 0.119 \\
\hline \multicolumn{4}{|c|}{ Fasting insulin (mIU/L) } \\
\hline$<20$ & Reference & 1.00 & - \\
\hline$\geq 20$ & $1.96-38.9$ & 5.74 & 0.008 \\
\hline \multicolumn{4}{|l|}{ HOMA-IR } \\
\hline$<2.66$ & Reference & 1.00 & - \\
\hline$\geq 2.66$ & $2.58-73.2$ & 10.06 & 0.003 \\
\hline \multicolumn{4}{|c|}{ Total cholesterol (mmol/L) } \\
\hline$<5.69$ & Reference & 1.00 & - \\
\hline$\geq 5.69$ & $0.93-2.86$ & 1.98 & 0.047 \\
\hline \multicolumn{4}{|l|}{ LDL-C (mmol/L) } \\
\hline$<3.1$ & Reference & 1.00 & - \\
\hline$\geq 3.1$ & $1.88-7.43$ & 2.23 & 0.035 \\
\hline \multicolumn{4}{|l|}{ HDL-C (mmol/L) } \\
\hline$<1.55$ & Reference & 1.00 & - \\
\hline$\geq 1.55$ & $0.25-0.89$ & 0.37 & 0.024 \\
\hline \multicolumn{4}{|c|}{ Triglycerides (mmol/L) } \\
\hline$<1.47$ & Reference & 1.00 & - \\
\hline$\geq 1.47$ & $1.03-2.96$ & 1.38 & 0.065 \\
\hline \multicolumn{4}{|l|}{ Apo A (g/L) } \\
\hline$<1.61$ & Reference & 1.00 & - \\
\hline$\geq 1.61$ & $0.63-2.24$ & 1.48 & 0.223 \\
\hline \multicolumn{4}{|l|}{ Apo B (g/L) } \\
\hline$<1.05$ & Reference & 1.00 & - \\
\hline$\geq 1.05$ & $0.56-2.17$ & 1.25 & 0.446 \\
\hline \multicolumn{4}{|l|}{ hs-CRP (mg/L) } \\
\hline$<3$ & Reference & 1.00 & - \\
\hline$\geq 3$ & $1.85-6.37$ & 2.18 & 0.038 \\
\hline
\end{tabular}

the incidence of cardiovascular disease in vitamin D-deficient women with PCOS by improving dyslipidaemia. However, the relationship between serum $25(\mathrm{OH}) \mathrm{D}$ and triglyceride concentration in PCOS women is controversial according to 
previous studies (28). This study demonstrated that no significant correlation was found between serum $25(\mathrm{OH}) \mathrm{D}$ concentration and triglycerides, Apo A or Apo B, which was consistent with the results of Li et al. (12).

Normal metabolism of insulin is affected by activation of inflammatory pathways. According to the published studies, the positive effects of vitamin $\mathrm{D}$ on sugar metabolism including regulation of insulin secretion and inhibition of proinflammatory cytokines (29). The data in our study displayed that serum $25(\mathrm{OH}) \mathrm{D}$ concentration in women with PCOS was significantly negatively correlated with hs-CRP. Results from a randomized controlled trial showed that vitamin D treatment significantly decreased serum hs-CRP in PCOS women with vitamin D deficiency (30). In addition, serum hs-CRP was improved by high dose vitamin D supplementation among PCOS women with IR (17).

Clinical manifestations of PCOS include obesity, metabolic syndrome and chronic inflammation, which are related to vitamin D deficiency $(10-14,29)$. Our findings revealed that serum 25(OH)D level in PCOS women was significantly negatively correlated with BMI, WHR, fasting insulin, HOMAIR, total cholesterol, LDL-C, and hs-CRP. Additionally, serum $25(\mathrm{OH}) \mathrm{D}$ concentration was significantly positively correlated with HDL-C. Furthermore, the data from logistic regression model of risk factors for vitamin $\mathrm{D}$ deficiency revealed that increased BMI and WHR, high levels of fasting insulin, HOMAIR, total cholesterol, LDL-C, and hs-CRP were regarded as risk factors of vitamin D deficiency in PCOS women. In comparison, outdoor exercise ( $\geq 1$ times daily) and high level of HDL-C were considered to be protective factors of vitamin $\mathrm{D}$ deficiency in PCOS women. These results suggest that PCOS women with metabolic risk factors are more likely to accompany vitamin $\mathrm{D}$ deficiency. Therefore, scholars from various countries have conducted randomized controlled trials on whether vitamin D supplementation can improve the metabolic risk factors of PCOS women. However, the results have been discordant. Vitamin D treatment improved the follicular development, menstrual cycle regulation, insulin resistance, and hyperandrogenism (21). However, Xue et al. showed that vitamin D supplementation did not change the HOMA-IR, LDL, DHEAS, free testosterone, and total testosterone in women with PCOS (31). The different results may be related to sample size, vitamin D supplement dose, participant habits, and place of residence. Therefore, multi-center randomized controlled trials with large sample sizes are needed

\section{REFERENCES}

1. March WA, Moore VM, Willson KJ, Phillips DI, Norman RJ, Davies MJ. The prevalence of polycystic ovary syndrome in a community sample assessed under contrasting diagnostic criteria. Hum Reprod. (2010) 25:544-51. doi: 10.1093/humrep/d ep399

2. The Amsterdam ESHRE/ASRM-Sponsored 3rd PCOS Consensus Workshop Group. Consensus on women's health aspects of polycystic ovary syndrome (PCOS). Hum Reprod. (2012) 27:14-24. doi: 10.1093/humrep/der396

3. Lim SS, Davies MJ, Norman RJ, Moran LJ. Overweight, obesity and central obesity in women with polycystic ovary syndrome: a systematic to explore the metabolic role of vitamin $\mathrm{D}$ supplementation in women with PCOS.

This study has some limitations. The sample size of the participants was relatively small. In addition, this was a singlecenter study of PCOS women in the city of Xi'an in Shaanxi, China. Hence, the results of this research needs to be proved through multi-center surveys with large sample sizes in other areas in China.

\section{CONCLUSION}

Our data show that vitamin D deficiency is prevalent in PCOS women in Shaanxi China, especially in those with obesity and IR. The serum $25(\mathrm{OH}) \mathrm{D}$ concentration was correlated with metabolic risk factors in PCOS women. Multi-center randomized controlled trials with large sample sizes are needed to explore the metabolic role of vitamin D supplementation in women with PCOS.

\section{DATA AVAILABILITY STATEMENT}

All datasets generated for this study are included in the article/supplementary material.

\section{ETHICS STATEMENT}

The studies involving human participants were reviewed and approved by The First Affiliated Hospital of Xi'an Jiaotong University Institutional Review Board. The patients/participants provided their written informed consent to participate in this study.

\section{AUTHOR CONTRIBUTIONS}

LW and XY conceived the study and wrote the manuscript. SL and FL collated data. XY and EB analyzed data. All authors have given final approval of the article to be published.

\section{FUNDING}

This study was supported by the basic research program of natural science of Shaanxi province, China (2019JM-569); the institutional foundation of the first affiliated hospital of Xi'an Jiaotong university (2019ZYTS-03). 
systematic review and meta-analysis. Hum Reprod Update. (2010)16:347-63. doi: 10.1093/humupd/dmq001

7. Wild RA, Rizzo M, Clifton S, Carmina E. Lipid levels in polycystic ovary syndrome: systematic review and meta-analysis. Fertil Steril. (2011)95:10739.e1-11. doi: 10.1016/j.fertnstert.2010.12.027

8. Bizzaro G, Antico A, Fortunato A, Bizzaro N. Vitamin D and autoimmune diseases: is vitamin D receptor (VDR) polymorphism the culprit? Isr Med Assoc J. (2017) 19:438-43. doi: 10.4172/2168-9849.1000e105

9. Mohammad AM, Shammo NA, Jasem JA. Vitamin D status in acute myocardial infarction: a case-control study. Cardiovasc Endocrinol Metab. (2018) 7:93-6. doi: 10.1097/XCE.0000000000000160

10. Skaaby $\mathrm{T}$, Thuesen BH, Linneberg A. Vitamin D, cardiovascular disease and risk factors. Adv Exp Med Biol. (2017) 996:221-30. doi: 10.1007/978-3-319-56017-5_18

11. Sassi F, Tamone C, D'Amelio P. Vitamin D: nutrient, hormone, and immunomodulator. Nutrients. (2018) 10:E1656. doi: 10.3390/nu10111656

12. Li HW, Brereton RE, Anderson RA, Wallace AM, Ho CK. Vitamin $\mathrm{D}$ deficiency is common and associated with metabolic risk factors in patients with polycystic ovary syndrome. Metabolism. (2011) 60:1475-81. doi: 10.1016/j.metabol.2011.03.002

13. Joham AE, Teede HJ, Cassar S, Stepto NK, Strauss BJ, Harrison CL, et al. Vitamin D in polycystic ovary syndrome: relationship to obesity and insulin resistance. Mol Nutr Food Res. (2016) 60:110-18. doi: $10.1002 / \mathrm{mnfr} .201500259$

14. Ng BK, Lee CL, Lim PS, Othman H, Ismail NAM. Comparison of 25hydroxyvitamin D and metabolic parameters between women with and without polycystic ovarian syndrome. Horm Mol Biol Clin Investig. (2017) 31:j/hmbci.2017.31. doi: 10.1515/hmbci-2016-0057

15. Chen ZJ, Shi Y, Sun Y, Zhang B, Liang X, Cao Y, et al. Fresh versus frozen embryos for infertility in the polycystic ovary syndrome. N Engl J Med. (2016) 375:523-33. doi: 10.1056/NEJMoa1513873

16. Li L, Zhang F, Cui J, Shi Y, Xiang J, Wang X, et al. Association of betatrophin with metabolic characteristics in overweight/obese and lean women with PCOS. Gynecol Endocrinol. (2017) 33:238-43. doi: 10.1080/09513590.2016.1252743

17. Jamilian M, Foroozanfard F, Rahmani E, Talebi M, Bahmani F, Asemi Z. Effect of two different doses of vitamin D supplementation on metabolic profiles of insulin-resistant patients with polycystic ovary syndrome. Nutrients. (2017) 9:E1280. doi: 10.3390/nu9121280

18. Yun CF, Chen J, He Y, Mao D, Wang R, Zhang Y, et al. Vitamin D deficiency prevalence and risk factors among pregnant Chinese women. Public Health Nutr. (2017) 20:1746-54. doi: 10.1017/S1368980015002980

19. Holick MF. The vitamin D deficiency pandemic: approaches for diagnosis, treatment and prevention. Rev Endocr Metab Disord. (2017) 18:153-65. doi: 10.1007/s11154-017-9424-1

20. Thomson RL, Spedding S, Buckley JD. Vitamin D in the aetiology and management of polycystic ovary syndrome. Clin Endocrinol. (2012) 77:34350. doi: 10.1111/j.1365-2265.2012.04434.x

21. Fang F, Ni K, Cai Y, Shang J, Zhang X, Xiong C. Effect of vitamin D supplementation on polycystic ovary syndrome: a systematic review and meta-analysis of randomized controlled trials. Complement Ther Clin Pract. (2017) 26:53-60. doi: 10.1016/j.ctcp.2016.11.008

22. Krul-Poel YHM, Koenders PP, Steegers-Theunissen RP, Boekel ET, TerWee $\mathrm{MM}$, Louwers $\mathrm{Y}$ et al. Vitamin $\mathrm{D}$ and metabolic disturbances in polycystic ovary syndrome(PCOS): a cross-sectional study. PLoS ONE. (2018) 13:e0204748. doi: 10.1371/journal.pone.0204748

23. He CL, Lin ZM, Robb SW, Ezeamama AE. Serum vitamin D levels and polycystic ovary syndrome:a systematic review and meta-analysis. Nutrients. (2015)7:4555-77. doi: 10.3390/nu7064555

24. Muscogiuri G, Policola C, Prioletta A, Sorice G, Mezza T, Lassandro A, et al. Low levels of 25(OH)D and insulin-resistance: 2 unrelated features or a cause-effect in PCOS? Clin Nutr. (2012)31:476-80. doi: 10.1016/j.clnu.2011. 12.010

25. Teegarden D, Donkin SS. Vitamin D: emerging new roles in insulin sensitivity. Nutr Res Rev. (2009)22:82-92. doi: 10.1017/S09544224093 89301

26. Pittas AG, Lau J, Hu FB, Dawson-Hughes B. The role of vitamin D and calcium in type 2 diabetes: a systematic review and meta-analysis. $J$ Clin Endocrinol Metab. (2007) 92:2017-29. doi: 10.1210/jc.2007-0298

27. Wehr E, Pilz S, Schweighofer N, Giuliani A, Kopera D, Pieber TR, et al. Association of hypovitaminosis D with metabolic disturbances in polycystic ovary syndrome. Eur J Endocrinol. (2009) 161:575-82. doi: 10.1530/EJE-09-0432

28. Yildizhan R, Kurdoglu M, Adali E, Kolusari A, Yildizhan B, Sahin HG, et al. Serum 25-hydroxyvitamin D concentrations in obese and non-obese women with polycystic ovary syndrome. Arch Gynecol Obstet. (2009) 280:559-63. doi: 10.1007/s00404-009-0958-7

29. Chagas CE, Borges MC, Martini LA, Rogero MM. Focus on vitamin D, inflammation and type 2 diabetes. Nutrients. (2012) 4:52-67. doi: $10.3390 /$ nu4010052

30. Maktabi M, Chamani M, Asemi Z. The effects of vitamin D supplementation on metabolic status of patients with polycystic ovary syndrome: a randomized, double-blind, placebo-controlled trial. Horm Metab Res. (2017) 49:493-8. doi: 10.1055/s-0043-107242

31. Xue YP, Xu P, Xue K, Duan X, Cao J, Luan T, et al. Effect of vitamin $\mathrm{D}$ on biochemical parameters in polycystic ovary syndrome women: a meta-analysis. Arch Gynecol Obstet. (2017) 295:487-96. doi: $10.1007 / \mathrm{s} 00404-016-4247-y$

Conflict of Interest: The authors declare that the research was conducted in the absence of any commercial or financial relationships that could be construed as a potential conflict of interest.

Copyright (C) 2020 Wang, Lv, Li, Yu, Bai and Yang. This is an open-access article distributed under the terms of the Creative Commons Attribution License (CC BY). The use, distribution or reproduction in other forums is permitted, provided the original author(s) and the copyright owner(s) are credited and that the original publication in this journal is cited, in accordance with accepted academic practice. No use, distribution or reproduction is permitted which does not comply with these terms. 